\title{
Imágenes fotoacústicas para diagnósticos médicos
}

\author{
Carolyn L. Bayer ${ }^{A}$, Geoffrey P. Luke ${ }^{B}$, Stanislav Y. Emelianov ${ }^{C}$ \\ ${ }^{\text {A }}$ Departamento de Ingeniería Biomédica \\ ${ }^{\text {B }}$ Departamento de Ingeniería Eléctrica y Computación \\ ${ }^{\mathrm{C}}$ Departamento de Ingeniería Biomédica y Computación \\ Universidad de Texas en Austin Austin, Texas 78712 \\ carolynb@tulane.edu
}

\section{RESUMEN}

Este artículo presentan algunos de los aspectos más sobresalientes de la investigación y desarrollo de la acústica subacuática, así como reconoce el mérito de miembros de la Sociedad Americana de Acústica (Acoustics Society of America, ASA), quienes contribuyeron con su trabajo durante el periodo de la Segunda Guerra Mundial. Los resultados de tal trabajo se convirtieron en logros científicos y de ingeniería que aparecen en literatura sobre acústica.

\section{PALABRAS CLAVE}

Sociedad Americana de Acústica, sonar, detección submarina.

\section{ABSTRACT \\ This paper presents some of the highlights about research and development of sub-aquatic acoustics, as well as it gives recognition to the members of the Acoustical Society of America (ASA), who contributed with their work along the Second World War. The results of such work have turned into scientific and engineering achievements, which are published in literature about acoustics.}

\section{KEYWORDS}

Acoustic Society of America, sonar, submarine detection.

\section{INTRODUCCIÓN}

La imagen fotoacústica tiene el potencial de proporcionar el diagnóstico no invasivo de numerosas enfermedades frecuentes, en tiempo real, debido a la capacidad única de la tecnología para visualizar cambios moleculares profundos dentro del tejido vivo con resolución espacial comparable al ultrasonido. La imagen fotoacústica es una técnica de imagen híbrida que combina la capacidad de contraste y la sensibilidad espectral de la imagen óptica con la resolución y la capacidad de penetración en el tejido del ultrasonido. Durante el proceso de obtención de imágenes fotoacústicas, los materiales absorben la energía de la luz y convierten la luz en calor mediante la relajación no radiativa. Cuando los materiales se calientan, se expanden en tamaño debido a sus propiedades termoelásticas, que generan una onda de presión. Estas ondas pueden propagarse a través del entorno circundante para ser detectadas en la superficie. Este efecto es familiar para cualquiera que haya experimentado una tormenta de verano, un 
rayo calienta rápidamente el aire, resultando en que éste se expande y genera un trueno audible. En general, el calentamiento induce la expansión del material (por ejemplo el efecto termoacústico) podría ser causado por muchas formas de transferencia de energía, pero el término "fotoacústica" especifica la conversión de luz en calor, resultando en la generación de ondas sonoras características.

El efecto fotoacústico fue descubierto por primera vez por Alexander Graham Bell en 1880. ${ }^{1}$ A partir de sus experimentos dedujo que una luz brillante intermitente podría calentar ópticamente materiales absorbentes, causando la expansión del material de una manera que genere ondas vibratorias audibles. Bell demostró que las fibras más oscuras producen sonidos más fuertes que las fibras más claras, un principio que es consistente con la relación fotoacústica general en uso hoy en día, la amplitud de la señal fotoacústica generada es proporcional a la cantidad de luz absorbida. Bell también mostró, al separar la luz blanca con un prisma, ciertas combinaciones de color de la luz y las fibras podrían generar un sonido más fuerte. Hoy la imagen fotoacústica de longitudes de onda múltiples utiliza este mismo principio, cambiando la longitud de onda de la luz y correlacionando la amplitud de la respuesta fotoacústica con los espectros de absorción de los materiales que se están fotografiando.

Una aplicación moderna del efecto fotoacústico es la generación de imágenes médicas de cromóforos biológicos típicamente presentes en el tejido, que pueden absorber la energía de la luz resultando en la generación de transitorios fotoacústicos. Las ondas de presión fotoacústica pueden ser recibidas por transductores de ultrasonido en la superficie externa del tejido, haciendo que la imagen fotoacústica sea un método de imagen médica no invasivo y no ionizante capaz de resolución similar al ultrasonido, a una profundidad de tejido significativa. Las imágenes médicas fotoacústicas se propusieron por primera vez a mediados de la década de $1990,{ }^{2,3}$ y los reportes iniciales sobre el uso del efecto fotoacústico para imágenes de animales vivos se publicaron nueve años después. Hoy existen muchas demostraciones in vivo de imágenes fotoacústicas de aplicaciones biomédicas relevantes para el diagnóstico médico, incluyendo cáncer, ${ }^{5,6}$ función vascular cerebral, ${ }^{7-9}$ y cardiovasculares, ${ }^{10}$ e ingeniería de soporte de tejido, ${ }^{11,12}$ impulsando avances traslacionales en imagen clínica fotoacústica. ${ }^{13}$

Mientras que los métodos de imagen médica existentes, incluyendo el ultrasonido, son capaces de producir imágenes de lo que está debajo de la piel, la mayoría de ellos proporcionan contraste entre las características anatómicas dentro del tejido. Por ejemplo, la diferencia en la impedancia acústica entre el tejido blando y un tumor proporcionan contraste dentro de una imagen de ultrasonido. Aunque la anatomía es crítica para entender la imagen, en muchas enfermedades la anatomía por sí sola no se puede utilizar para dar un diagnóstico determinado de manera concluyente. En cambio, las propiedades fisiológicas y bioquímicas del sistema influyen en la progresión de la enfermedad y, por tanto, en el pronóstico del paciente. Se requieren capacidades de imagen funcional para proporcionar información fisiológica, mientras que la información bioquímica puede ser proporcionada por imágenes moleculares. En comparación con el ultrasonido, la imagen fotoacústica proporciona capacidades mejoradas para la imagen funcional y molecular. Por ejemplo, la saturación de oxígeno en la sangre, una importante propiedad funcional relevante para muchos procesos de 
enfermedad, puede evaluarse mediante fotoacústica. ${ }^{7}$ La imagen fotoacústica también puede proporcionar información molecular mediante el uso de una sonda o un trazador, que puede ser utilizada para generar el contraste necesario para producir una imagen. ${ }^{14}$ Debido al potencial para realizar imágenes funcionales y moleculares in vivo no invasivas en tiempo real, la imagen fotoacústica se está aplicando cada vez más como método clínico y preclínico destinado a mejorar el diagnóstico médico.

\section{ANTECEDENTES}

En un sistema de imagen fotoacústica contemporáneo, se utiliza un láser pulsado de nanosegundos para generar ondas de presión transitoria, que son recibidas por un transductor de ultrasonido y procesada para formar imágenes de tejido biológico (figura 1).

En una configuración típica, el pulso de luz emitido por el láser va acompañado de una señal de disparo enviada al hardware del sistema de imagen, coordinando

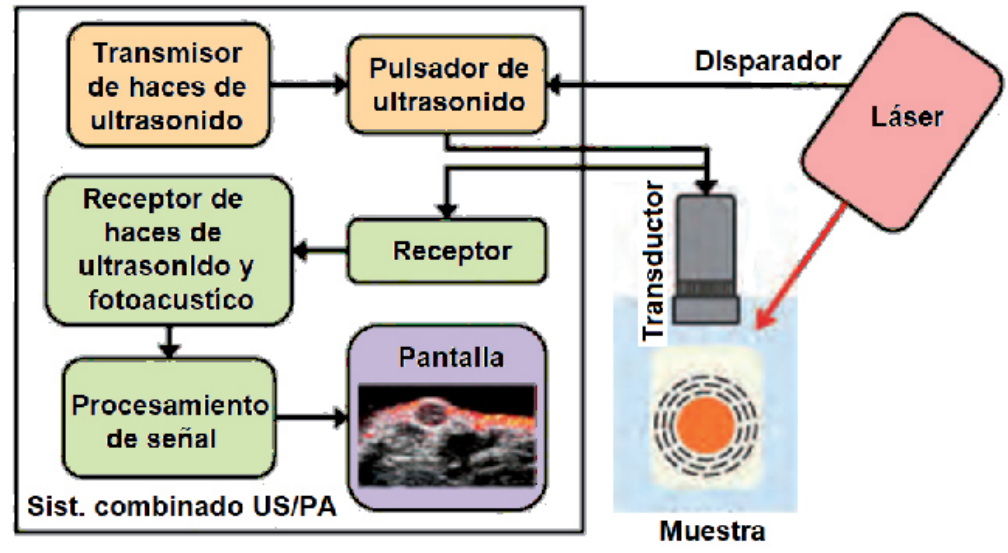

US-Ultrasonido; PA-Fotoacústica

Fig. 1. Instrumentación y componentes principales de procesamiento que comprenden un sistema combinado de ultrasonido y fotoacústica. La señal fotoacústica, generada por la absorción óptica de energía láser por la muestra, se recibe por un transductor de ultrasonido, formada y procesada para formar una imagen fotoacústica. El transductor emite el ultrasonido, adecuadamente formado por haces, y el ultrasonido reflejado es recibido, formado por rayos y procesado para formar la imagen de ultrasonido.

la adquisición de la señal fotoacústica. Después de la emisión del pulso láser, las señales fotoacústicas generadas por los absorbentes ópticos dentro del campo de visión son recogidas por el transductor (figura 2). Al igual que con el ultrasonido, el momento en que se recibe la señal fotoacústica está relacionado con la profundidad del objeto absorbente dentro de la muestra por la velocidad del sonido en el tejido.

Los sistemas multimodales, combinando ultrasonido y fotoacústica, aplican un retardo de tiempo ( $\tau \mathrm{PA}$ ) después de la señal de disparo láser, como se muestra en la figura 2, después del cual la señal de ultrasonido es transmitida y recibida para ser procesada utilizando técnicas usuales. Debido a las similitudes en el hardware del sistema y el diseño de procesamiento entre la imagen fotoacústica 


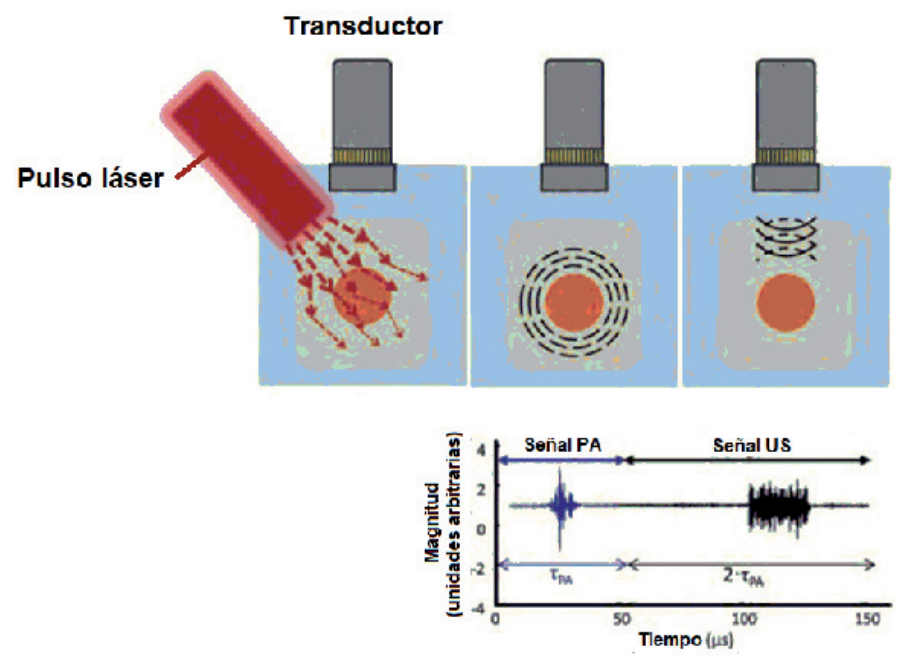

Fig. 2. Generación de señales fotoacústicas y ultrasónicas. (a) El pulso láser es dispersado y absorbido por la muestra. (b) La absorción que conduce al calentamiento genera una onda de presión transitoria que es recibida por el transductor. (c) Después de un retraso, TPA, el ultrasonido se transmite y recibe durante el período de tiempo 2 - TPA.

y la ecografía, un sistema de ultrasonido existente puede ser modificado para adquirir señales fotoacústicas sin más esfuerzo que mediante la adición de una fuente de luz pulsada, realizando modificaciones menores de hardware para programar adecuadamente las señales transmitidas y recibidas, y modificar la interfaz de usuario del software y los métodos de procesamiento de señales. Varias compañías están comercializando sistemas fotoacústicos integrados para imágenes de animales pequeños, mostrados en la figura 3 , y es probable que pronto entren más en el mercado (dato de 2012). Estos sistemas se dividen en dos categorías diseñadas para utilizar un escáner de ultrasonido lineal traducible con el formado del haz de retardo y suma, o un conjunto fijo de transductores con reconstrucción de imágenes de tomografía computarizada.

Los sistemas de escaneo fotoacústico lineal producen una visión bidimensional (2D) de una sola proyección, y luego se escanean en una tercera dimensión, un método de imagen volumétrica análogo a los sistemas de ultrasonido clínicos no invasivos de uso común, donde se barre manualmente a lo largo de la superficie del tejido con un arreglo de transductores. Los sistemas tomográficos utilizan una serie de transductores, a menudo colocados en un anillo alrededor del objeto que se está fotografiando, y luego usan algoritmos de reconstrucción para reproducir la imagen. Visual Sonics Inc. comercializa el Vevo LAZR (Figura 3a), que utiliza su pequeña plataforma de ultrasonido animal de alta resolución (Vevo 2100) en combinación con un láser pulsado nanosegundo sintonizable. Cuando la imagen de cuerpo entero es una prioridad, el sistema de tomografía optoacoustica multiespectral (Figura 3b), producido por iThera Medical, guía el haz láser para iluminar un anillo de luz alrededor del animal que está siendo fotografiado, y luego se mueve en la tercera dimensión para adquirir imágenes volumétricas. ${ }^{15}$ El Nexus 128 (Figura 3c), producido por Endra, proporciona un sistema fotoacústico tomográfico alternativo. Todos estos sistemas consisten en una fuente de láser sintonizable que proporciona luz infrarroja cercana (NIR) aproximadamente entre 680 y $970 \mathrm{~nm}$. La elección de un sistema de exploración 


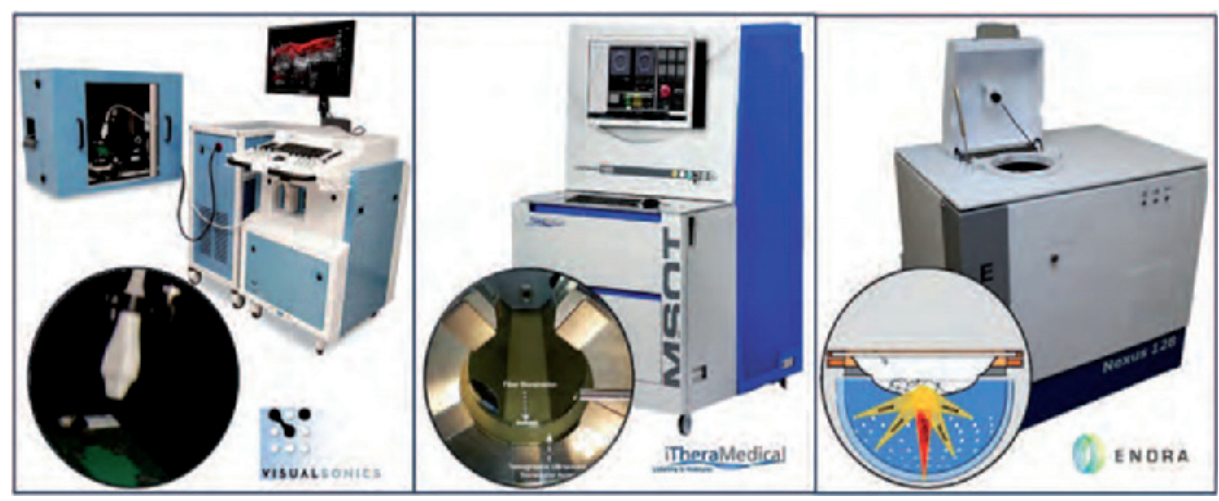

Fig. 3. Sistemas de imagen fotoacústica preclínica disponibles en el mercado.

o tomografía depende en gran medida de la aplicación. El usuario debe considerar la resolución alcanzable, que depende de la frecuencia central y el ancho de banda del transductor, el formador del haz o algoritmos de reconstrucción implementada, y también la sensibilidad alcanzable, que está influenciado por la fluencia de la luz y la distribución entregada dentro del tejido. Un sistema de escaneo por ultrasonido generalmente será más flexible, acomodando una variedad de tipos de animales, y potencialmente proporcionando múltiples matrices de transductores optimizados para diferentes profundidades de escaneo. Un sistema de imagen fotoacústica basado en una plataforma de escáner de ultrasonido proporciona técnicas adicionales de imagen funcional (como el modo Doppler de ultrasonido) para proporcionar información anatómica y funcional. En contraste, los sistemas tomográficos son capaces de adquirir datos volumétricos en un tiempo más corto, y tienen menos ruido en forma de mota fotoacústica debido a la distribución espacial del anillo transductor. Sin embargo, las aplicaciones clínicas de un sistema de tomografía fotoacústica serán limitadas, con una notable excepción; la imagen tomográfica fotoacústica del tejido mamario. ${ }^{16}$

Mientras que los absorbentes ópticos que se encuentran de forma natural en el cuerpo, como la hemoglobina, los lípidos y la melanina, pueden utilizarse para generar imágenes ópticas, el uso de agentes de contraste de imagen de tamaño nanométrico permiten que la imagen fotoacústica se vuelvan verdaderamente "molecular" en el metodo de imagen in vivo. ${ }^{17,18} \mathrm{El}$ contraste fotoacústico puede mejorarse mediante el uso de fragmentos moleculares o nanopartículas sintetizadas químicamente. Las nanopartículas inorgánicas son de especial interés, debido a su flexibilidad con respecto a la forma, tamaño y química de la superficie. Estas características se pueden optimizar para mejorar la entrega de las nanopartículas al tejido de interés, como los tumores cancerosos. ${ }^{19} \mathrm{Si}$ la superficie de nanopartículas es modificada mediante la adición de un medio capaz de ser dirigido, estos agentes se vuelven agentes de imagen molecular, dado que las nanopartículas blanco deberían retenerse preferentemente dentro de la región del tejido que expresa el objetivo. Además, las nanopartículas metálicas de oro o plata, hay varios ejemplos, que se muestran en la figura 4, tienen resonancia de plasmón superficial, lo que significa que los electrones de valencia de los átomos oscilan colectivamente a una frecuencia característica. 


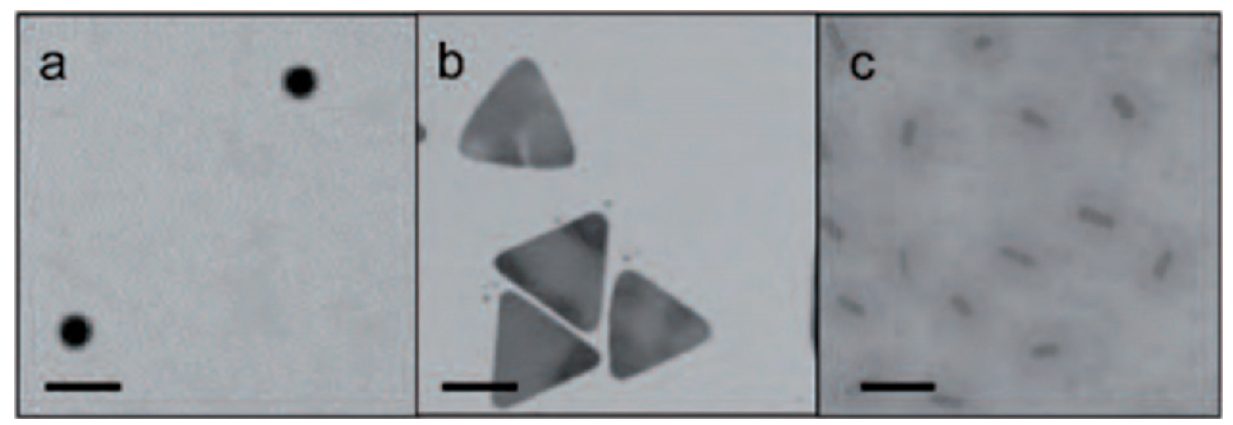

Fig. 4. Imágenes de microscopía electrónica de transmisión de nanopartículas metálicas plasmónicas que se han utilizado para la obtención de imágenes fotoacústicas in vivo. (a) Nanoesferas de oro; b) Nanoplacas de plata; c) Nanovarillas de oro recubiertas de sílice. Barras de escala $=100 \mathrm{~nm}$.

Cuando la luz incidente está en la misma frecuencia que la resonancia de plasma de superficie, esa luz es absorbida muy eficientemente y convertida en calor, haciendo de las nanopartículas metálicas excelentes agentes de contraste para la imagen fotoacústica. Las nanopartículas que se dirigen molecularmente se pueden arreglar para su entrega a tejidos particulares en función del tamaño, la forma y las propiedades de la superficie, mientras que la alta absorción óptica de nanopartículas metálicas genera una fuerte señal fotoacústica y proporciona un alto contraste con el tejido circundante. Mediante el uso de nanopartículas metálicas, también podemos ajustar sus propiedades de absorción óptica para aprovechar la "ventana óptica de tejido". Dentro de esta región de longitud de onda, entre aproximadamente $600 \mathrm{~nm}$ a $1300 \mathrm{~nm}$, la absorción óptica del tejido nativo es relativamente baja, por lo que la luz puede penetrar más profundamente en el tejido, permitiendo la adquisición de imágenes fotoacústicas a profundidades de tejido significativamente mayores. Mediante imágenes en longitudes de onda que no son altamente absorbidas en los tejidos, el efecto fotoacústico puede utilizarse para generar imágenes moleculares de alta resolución in vivo a una profundidad de tejido significativa. Sigue habiendo dudas sobre la seguridad de las nanopartículas metálicas para su uso en el cuerpo humano. ${ }^{20,21}$ De hecho, todavía se desconoce mucho sobre su toxicidad a corto y a largo plazo, pero muchos grupos están estudiando estos efectos, ${ }^{21} \mathrm{y}$ tres estudios clínicos de metodologías basadas en nanopartículas de oro para el tratamiento del cáncer están en marcha o se han completado. ${ }^{22}$ Además, la obtención de imágenes fotoacústicas podría contribuir a mejorar la comprensión de los mecanismos de biodistribución y aclaramiento que afectan a la toxicidad de las nanopartículas.

\section{DIAGNÓSTICO DE IMÁGENES FOTOACÚSTICAS}

En nuestros estudios, la información anatómica, funcional y molecular se adquirió utilizando una combinación de ultrasonido y de imágenes multilongitud de onda de ratones in vivo portadores de tumores cancerosos. Todos los métodos siguen protocolos aprobados por el Comité Institucional de Cuidado y Uso de Animales de la Universidad de Texas en Austin. Primero, desarrollamos un pequeño modelo animal de cáncer de mama que consistía en dos tumores establecidos a partir de líneas celulares de cáncer de mama humano con expresión de biomarcador de células diferenciales. 
Para este pequeño modelo animal, iniciamos tumores dentro de la almohadilla de grasa mamaria usando inyecciones de células cancerosas BT-474, que sobreexpresan el receptor celular HER2, y células cancerosas MDA-MB-231, que sobre-expresan la integrina $\alpha v \beta 3$, presente en la superficie celular de las células epiteliales de neovasculatura. Las nanovarillas de oro fueron elegidas como el agente de contraste molecular ya que, además de ser altamente absorbente, ópticamente, debido a los efectos de resonancia de plasma de superficie, sus espectros de absorción óptica pueden sintonizarse cambiando su relación de aspecto. ${ }^{23}$ Sintonizando nanovarillas para tener diferentes longitudes de onda de absorción óptica de pico, es posible distinguir entre múltiples agentes de contraste nanovarilla a través de imágenes fotoacústicas de longitudes de onda múltiples. ${ }^{24}$ Recubrimos las nanovarillas con sílice amorfa, lo que mejora la estabilidad térmica de la nanovarilla de oro ${ }^{25} \mathrm{y}$ la eficiencia de generación de señales fotoacústicas. ${ }^{26}$ Sílice-nanovarillas recubiertas con dos relaciones de aspecto diferentes fueron modificados químicamente para fijar anticuerpos de orientación, lo que permite que la nanopartícula sea absorbida preferentemente por las células tumorales. ${ }^{24}$ Los agentes de contraste se inyectaron en el torrente sanguíneo de un ratón que hacía crecer los tumores. Los agentes de contraste inyectados se distribuyen a través del sistema circulatorio, y se infiltran a través de los tejidos del ratón, incluyendo los tumores cancerosos.

La región tumoral del ratón fue fotografiada usando un escáner de ultrasonido animal pequeño de alta frecuencia Vevo 2100 (Visualsonics Inc.), integrado con un láser pulsado nanosegundo Spectraphysics Quantaray Pro Nd:YAG con un oscilador paramétrico óptico GWU Premiscan (OPO) para sintonizar la longitud de onda de la luz. Se utilizó un haz de fibra óptica para entregar la luz láser a ambos lados de un conjunto de transductores lineales. El sistema se utilizó para entregar entre $10-20 \mathrm{~mJ} / \mathrm{cm}^{2}$ de luz en un rango de longitudes de onda de $680-930 \mathrm{~nm}$. Un transductor de ultrasonido de $21 \mathrm{MHz}$ (MS250, Visualsonics Inc.) recogió las señales fotoacústicas en cada longitud de onda, seguido de la transmisión de ultrasonido y la recepción del ultrasonido reflejado por el mismo transductor, dando lugar a imágenes de ultrasonidos y fotoacústicas registradas conjuntamente. Se utilizó un motor de paso lineal para traducir la construcción del transductor y del haz de fibra, en pasos de $150 \mu \mathrm{m}$, para adquirir imágenes fotoacústicas y de ultrasonido en la tercera dimensión, promediando cuatro señales fotoacústicas en cada paso. El ultrasonido tridimensional (3D) y las imágenes fotoacústicas fueron capturadas en un volumen que rodeaba los dos tumores dentro de la almohadilla de grasa mamaria del ratón.

La amplitud de la señal fotoacústica recibida depende de la longitud de onda de la luz láser utilizada para iluminar la muestra. Esta dependencia puede ser sin mezclar en los espectros de absorción individuales de los absorbentes dentro del tejido oxyhemoglobina, deoxihemoglobina, nanopartículas 1 y nanopartículas 2. Se utilizó un método lineal de error espectral mínimo cuadrado en el que se suponía que cada voxel contenía una combinación de los cuatro absorbentes ópticos. ${ }^{27}$ En este método, la señal fotoacústica inicial, situado en una posición dentro de la imagen, depende de la concentración de los absorbentes en la región, la fluencia láser, y el parámetro Grüneisen. ${ }^{28}$ Los espectros de absorción óptica de hemoglobina se obtuvieron de la literatura ${ }^{29}$ y los espectros de nanopartículas se midieron utilizando UV-Espectroscopia de Vis (lector de 
microplacas Synergy HT, Biotek Instruments, Inc.). Si el problema espectral separa está sobreentrenado, que se logra adquiriendo datos fotoacústicos en más longitudes de onda que el número de absorbedores presentes en el tejido, entonces se puede obtener una estimación de error cuadrado medio mínimo para las concentraciones del absorbente. Las concentraciones estimadas de desoxihemoglobina, oxihemoglobina, nanopartículas 1 y nanopartículas 2 se muestran usando colormaps azul, rojo, verde y violeta, respectivamente. Para separación espectral, los datos fueron promediados en voxels de tamaño $500 \mu \mathrm{m}$ $200 \mu \mathrm{m} 300 \mu \mathrm{m}$. Las imágenes de los absorbedores ópticos fueron superpuestas en imágenes de ultrasonido en escala de grises para visualizar la anatomía en la región.

Se generaron imágenes anatómicas 3D de alta resolución de la región tumoral mediante el procesamiento de las señales de ultrasonido recibidas por el sistema integrado de ultrasonido y de imagen fotoacústica (figura 5). En la figura 5a, una imagen 3D de una sola longitud de onda de imagen fotoacústica, superpuesta en la imagen de ultrasonido, muestra claramente la anatomía dentro de la región de imágenes del ratón, incluyendo la vasculatura (en color, proporcionada por la imagen fotoacústica), y la capa de piel y el fémur dentro de la pierna (en escala de grises, proporcionada por la imagen de ultrasonido).En nuestro modelo de ratón podemos identificar las regiones tumorales mediante la localización de dos regiones hipoecoicas bajo la piel, como se muestra en la ecografía 2D en la figura 5 b. De la señal fotoacústica (figuras 5 c y $5 d$ ), está claro que hay absorbentes de luz dentro de la piel, absorción de luz de la vasculatura que rodea los tumores, y de los propios tumores. Si bien tanto el ultrasonido como las imágenes fotoacústicas indican la presencia de tumores, las imágenes anatómicas por sí solas no proporcionan información suficiente para diagnosticar la malignidad, ya que la precisión del ultrasonido en la distinción de tumores benignos y malignos es insuficiente. ${ }^{30}$
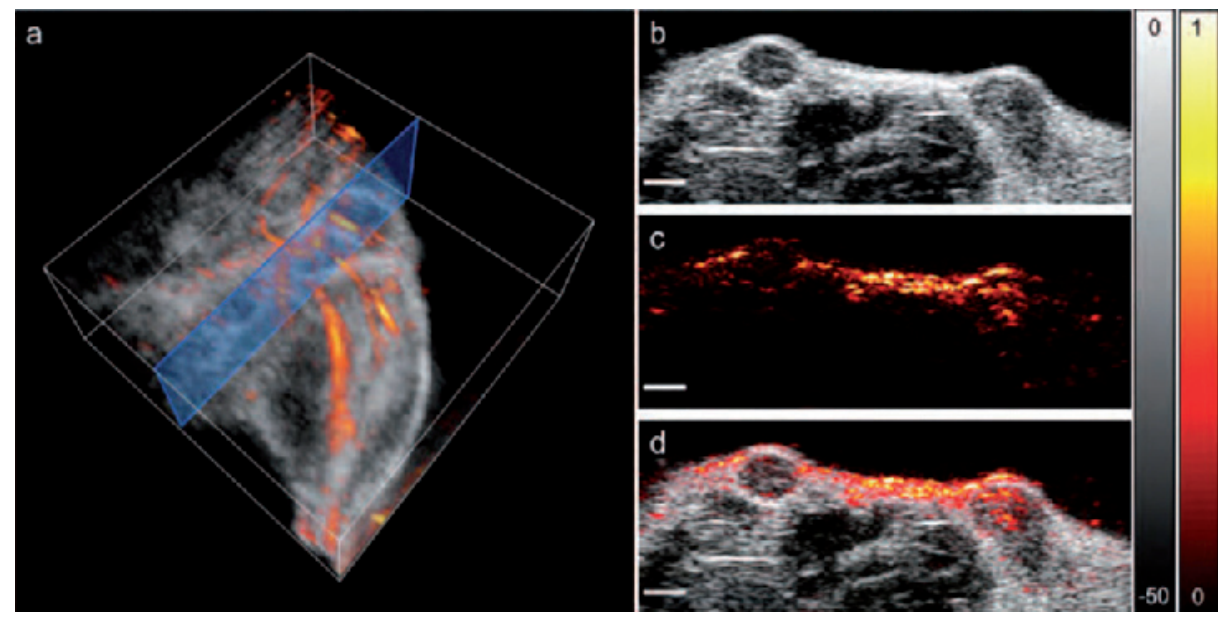

Fig. 5. Imágenes anatómicas fotoacústicas y ecográficas in vivo. (a) Superposición 3D de imágenes fotoacústicas y de ultrasonido de la parte superior de la pierna/ región abdominal. La caja azul muestra el plano de imagen 2D de (b-d). (b) Imagen ecográfica que muestra dos tumores hipoecoicos. (c) Imagen fotoacústica, adquirida utilizando una longitud de onda láser de $850 \mathrm{~nm}$. (d) Superposición de imágenes fotoacústicas y ultrasónicas. Barras de escala $=2 \mathrm{~mm}$. 
Mientras que el ultrasonido in vivo y las imágenes fotoacústicas de longitud de onda única en la figura 5 proporcionan información anatómica de alta resolución, se desconoce la composición celular del tumor, y por lo tanto somos incapaces, en esta etapa de la imagen, para identificar las características funcionales o la expresión molecular celular, que podría ayudar en la identificación de un tumor benigno canceroso.

Las imágenes fotoacústicas funcionales, por ejemplo la saturación de oxígeno en la sangre del tumor, podrían proporcionar criterios adicionales para evaluar la función tumoral. Adquirimos información funcional mediante la detección de señales fotoacústicas en múltiples longitudes de onda láser y la destrucción espectral de las señales como se describe anteriormente. Para la obtención de imágenes funcionales del modelo animal descrito anteriormente, adquirimos señales fotoacústicas generadas por luz láser entre $680-850 \mathrm{~nm}$. Como se muestra en la figura 6, podemos observar la saturación de oxígeno de la sangre dentro del tejido normal y dentro de las regiones tumorales. Las concentraciones estimadas de desoxihemoglobina y oxihemoglobina se muestran usando una escala de color que va desde azul ( $0 \%$ saturación de oxígeno) a rojo (100\% saturación de oxígeno). La imagen 3D que se muestra en la figura 6a indica la distinción del flujo venuo del flujo arterial dentro de la vasculatura del tejido de la imagen. Mientras que el estudio de la vasculatura tiene relevancia para algunos nichos de diagnósticos médicos, más significativamente, podemos utilizar la información funcional proporcionada por la imagen fotoacústica multilongitud de onda para estudiar la saturación de oxígeno en la sangre del tumor.

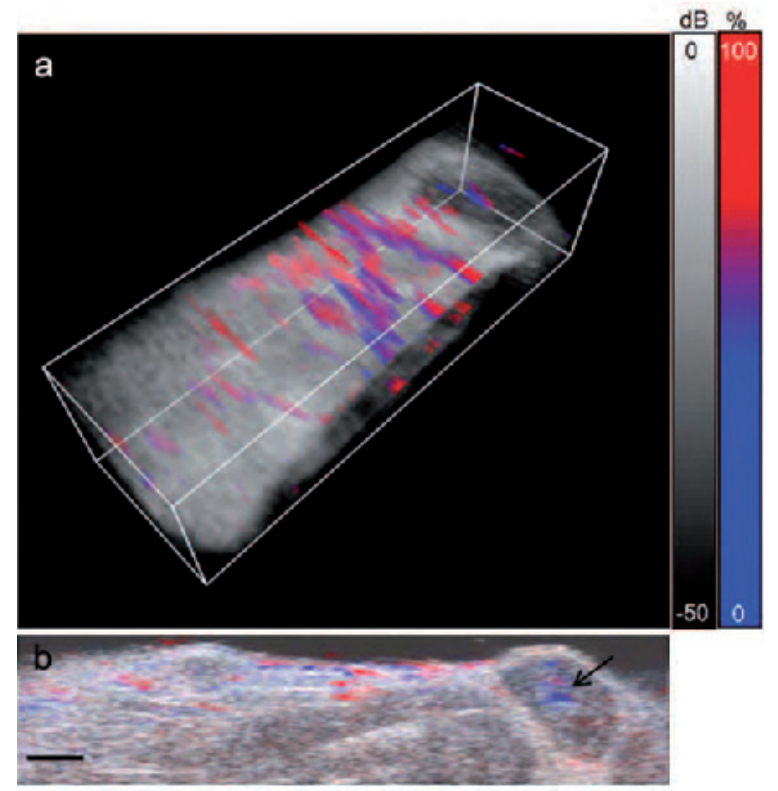

Fig. 6. Imagen funcional in vivo de la saturación de oxígeno en sangre. (a) Superposición $3 \mathrm{D}$ de porcentaje de saturación de oxígeno en sangre, determinada a partir de la destrucción espectral de la señal fotoacústica multilongitud de onda, y la imagen de ultrasonido. El volumen es de $23 \mathrm{~mm}$ (ancho) $\times 6 \mathrm{~mm}$ (alto) $\times 7 \mathrm{~mm}$ (dirección escaneada). (b) 2D rebanada del porcentaje de saturación de oxígeno en sangre y la señal de ultrasonido. La flecha negra indica una región hipóxica dentro de un tumor. Barras de escala $=2 \mathrm{~mm}$. 
Los tumores malignos hipóxicos tienen un pronóstico peor, lo que hace que la saturación de oxígeno en la sangre sea útil para la evaluación del tratamiento y la respuesta terapéutica. ${ }^{31}$ Como se muestra en la figura $6 \mathrm{~b}$, hay una región hipóxica dentro del núcleo del tumor fotografiado. Esta menor saturación de oxígeno es probable debido a la alta actividad metabólica de las células ubicadas dentro de esa región, lo que lleva a una entrega insuficiente de oxígeno dentro del tumor. La imagen fotoacústica funcional de la saturación de oxígeno en sangre podría utilizarse para estudiar la respuesta del tumor al tratamiento terapéutico, no invasivo, en tiempo real y durante largos períodos de crecimiento tumoral.

Por último, podemos seguir procesando las imágenes fotoacústicas para ver la señal que se correlaciona con los espectros de absorción óptica de los agentes de contraste inyectados. En los estudios que se muestran aquí, hemos utilizado nanovarillas de oro recubiertas de sílice, pero es importante señalar que una gran variedad de agentes de contraste se pueden utilizar con éxito para la imagen fotoacústica molecular; ${ }^{32}$ imágenes fotoacústicas in vivo con contraste mejorado se ha demostrado con el azul de metileno de color aprobado por la FDA,${ }^{33}$ con nanoesferas de oro, ${ }^{27} \mathrm{o}$ con nanoplacas de plata. ${ }^{34}$ Como se muestra en la figura 7, dentro de nuestro modelo de ratón, las regiones de acumulación de nanopartículas corresponden a las regiones tumorales identificadas con la ecografía y la imagen fotoacústica funcional de la saturación de oxígeno en sangre. Con el tiempo, hay un aumento en la señal atribuida a los agentes de contraste de nanopartículas dentro de la región tumoral (figuras $7 \mathrm{~b}$ y $7 \mathrm{c}$ ). La acumulación de nanopartículas podría deberse a dos efectos. Se sabe que los tumores tienen vasculatura con fugas, lo que permite una mayor entrega de partículas de tamaño nano a través del efecto mejorado de permeabilidad y retención. Además, las nanopartículas inyectadas fueron bioconyugadas a anticuerpos específicos para receptores celulares sobre expresados sobre tipos celulares utilizados para iniciar los tumores, por lo que es probable que el objetivo activo mejore la retención de las nanopartículas específicas dentro de la región tumoral. Este ejemplo demuestra cómo el uso de nanopartículas puede proporcionar información molecular sobre la expresión celular del tumor.

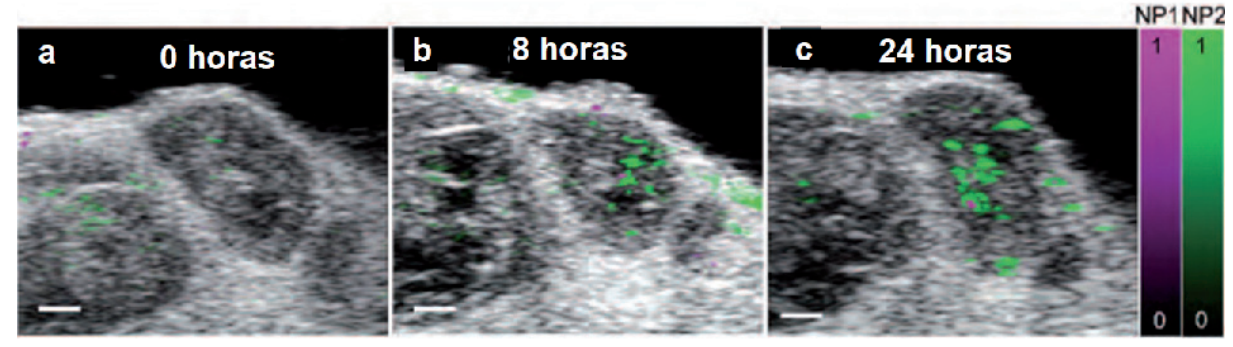

Fig. 7. Imagen molecular in vivo de la acumulación de agentes de contraste dentro de un tumor dirigido. La señal fotoacústica de longitudes de onda múltiples se mezcló en dos componentes correspondientes a dos agentes de contraste diferentes, nanopartículas 1 (NP1) y nanopartículas 2 (NP2). (a) Superposición 2D de imagen de ultrasonido, distribución de NP1 y NP2 antes de la inyección del agente de contraste, mostrando una señal fotoacústica de fondo mínima. La acumulación de NP1 y NP2 dentro del tumor se muestra (b) 8 horas y (c) 24 horas después de la inyección intravenosa. Barras de escala $=1 \mathrm{~mm}$. 
De esta manera, la fotoacústica combina los beneficios de la resolución óptica de alto contraste de las sondas de interacción luminosa, con las capacidades de profundidad de imagen del ultrasonido.

\section{CONCLUSIONES}

La fortaleza de la imagen fotoacústica está en su capacidad hacer imagenes de cambios funcionales y moleculares, a una profundidad de tejido significativa, en animales vivos. Los investigadores acaban de empezar a explotar las técnicas de imagen fotoacústica para emprendernuevos descubrimientos delas características funcionales y moleculares del cáncer, las enfermedades cardiovasculares y las enfermedades neurológicas. Concebimos la imagen fotoacústica como una herramienta crítica en el diagnóstico médico y la terapia guiada por imágenes en el futuro. Mientras que la mayoría de las aplicaciones de imagen funcional fotoacústica hasta ahora han utilizado fuentes de contraste que están presentes naturalmente, también es posible introducir agentes de contraste de activación bioquímica para la imagen funcional. Por ejemplo, existen muchos pigmentos que cambian su espectro de absorción óptica en base al $\mathrm{pH}$, o en presencia de una enzima. Al añadir un agente de contraste activable, podemos utilizar el cambio en la intensidad de la señal fotoacústica de longitudes de onda múltiples para proporcionar información cuantitativa sobre el entorno bioquímico dentro de los tejidos, y monitorear los cambios en este entorno.

Mientras que este artículo se centra en la imagen, también es de notar que existe una sinergia natural entre la imagen fotoacústica y la terapia. Por ejemplo, la terapia térmica se puede lograr mediante el uso de una fuente de luz continua para calentar los absorbedores ópticos utilizados para el contraste fotoacústico, a una temperatura que puede conducir a la destrucción de las células y el tejido circundante. ${ }^{35}$ Adicionalmente, Los agentes de contraste fotoacústicos multimodales pueden diseñarse para proporcionar la entrega de moléculas terapéuticas activadas por láser, por ejemplo, utilizando un recubrimiento de polímero sensible a la temperatura, ${ }^{36} \mathrm{o}$ utilizando el calor para liberar terapias covalentes. ${ }^{37} \mathrm{La}$ imagen fotoacústica no está exenta de limitaciones. Al igual que el ultrasonido, la resolución estará limitada físicamente por la relación inversa entre la profundidad de la imagen y la frecuencia del ultrasonido a medida que la frecuencia aumenta, la resolución también aumenta, sin embargo la atenuación de la señal es mayor en frecuencias más altas, lo que lleva a limitaciones en la profundidad de la imagen. La sensibilidad se ve afectada por la dispersión de la luz dentro del tejido, sin embargo nuevas técnicas para maximizar la señal al ruido, incluyendo agentes de contraste mejorados, y variando las características de la luz láser, pueden ayudar a minimizar la señal de fondo. Otras limitaciones incluyen que el tiempo requerido para escanear el lograr una alta resolución lateral depende de un haz estrecho, lo que significa que el transductor receptor (en sistemas de escaneo) o el objeto que está siendo fotografiado (en sistemas tomográficos) debe ser escaneado en una tercera dimensión para ampliar el campo de visión. Esta limitación de tiempo limita la resolución temporal de la información funcional y molecular que puede obtenerse a la escala de tiempo de minutos. Actualmente, sólo existen en el mercado sistemas preclínicos de obtención de imágenes fotoacústicas, pero la información funcional y molecular adicional disponible a través de esta modalidad de obtención de 
imágenes significará su creciente uso en estudios preclínicos, proporcionar los antecedentes de investigación necesarios para las adaptaciones clínicas.

\section{REFERENCIAS}

1. 1. A. G. Bell, "On the production and reproduction of sound by light," Am. J. Sci. 20, 305-324 (1880).

2. R. A. Kruger "Photoacoustic ultrasound," Med. Phys. 21, 127- 131 (1994).

3. A. A. Oraevsky, S. L. Jacques, R. O. Esenaliev, and F. K. Tittel "Time-resolved optoacoustic imaging in layered biological tissues," OSA Proc.Advances in Optical Imaging and Photon Migration 21, 161-165 (1994).

4. X. Wang, Y. Pang, G. Ku, X. Xie, G. Stoica, and L. V. Wang "Noninvasive laser-induced photoacoustic tomography for structural and functional in vivo imaging of the brain," Nature Biotechnol. 21, 803-806 (2003).

5. S. Mallidi, G. P. Luke, and S. Emelianov "Photoacoustic imaging in cancer detection, diagnosis, and treatment guidance," Trends in Biotechnol. 29, 213221 (2011).

6. J. Laufer, P. Johnson, E. Zhang, B. Treeby, B. Cox, B. Pedley, and P. Beard "In vivo preclinical photoacoustic imaging of tumor vasculature development and therapy," J. Biomed. Optics 17, 056016-1 (2012).

7. E. W. Stein, K. Maslov, and L. V. Wang "Noninvasive, in vivo imaging of blood-oxygenation dynamics within the mouse brain using photoacoustic microscopy, "J. Biomed. Optics 14, $020502-020502$ (2009).

8. J. Laufer, E. Zhang, G. Raivich, and P. Beard "Three-dimensional noninvasive imaging of the vasculature in the mouse brain using a high resolution photoacoustic scanner," Appl.Optics 48, D299-306 (2009).

9. V. Tsytsarev, K. I. Maslov, J. Yao, A. R. Parameswar, A. V. Demchenko, and L. V. Wang "In vivo imaging of epileptic activity using 2-nbdg, a fluorescent deoxyglucose analog," J. Neurosci. Methods 203, 136-140 (2012).

10.A. Taruttis, E. Herzog, D. Razansky, and V. Ntziachristos "Realtime imaging of cardiovascular dynamics and circulating gold nanorods with multispectral optoacoustic tomography," Optics Express 18, 19592-19602 (2010).

11.X. Cai, B. S. Paratala, S. Hu, B. Sitharaman, and L. V. Wang "Multiscale photoacoustic microscopy of single-walled carbon nanotube-incorporated tissue engineering scaffolds," Tissue Engineering Part C Methods 18, 310317 (2012).

12.S. Y. Nam, L. M. Ricles, L. J. Suggs, and S. Y. Emelianov "In vivo ultrasound and photoacoustic monitoring of mesenchymal stem cells labeled with gold nanotracers," PLoS ONE 7, e37267 (2012).

13.D. Piras, W. Xia, W. Steenbergen, T. van Leeuwen, and S. G. Manohar "Imaging breast lesions using the twente photoacoustic mammoscope: Ongoing clinical experience," Proc. SPIE 8223, 82231-82230C (2012).

14.G. P. Luke, D. Yeager, and S. Y. Emelianov "Biomedical applications of photoacoustic imaging with exogenous contrast agents," Ann. Biomed. Eng. 40, 422-437 (2012). 
Imágenes fotoacústicas para diagnósticos médicos / Carolyn L. Bayer, et al.

15.D. Razansky, A. Buehler, and V. Ntziachristos "Volumetric realtime multispectral optoacoustic tomography of biomarkers," Nature Protocols 6 , 1121-1129 (2011).

16.R. A. Kruger, R. B. Lam, D. R. Reinecke, S. P. Del Rio, and R. P. Doyle "Photoacoustic angiography of the breast," Med. Phys. 37, 6096-6100 (2010).

17.A. Agarwal, S. W. Huang, M. O’Donnell, K. C. Day, M. Day, N. Kotov, and S. Ashkenazi "Targeted gold nanorod contrast agent for prostate cancer detection by photoacoustic imaging," J. Appl. Phys. 102 (2007).

18.P.-C. Li, C.-R. C. Wang, D.-B. Shieh, C.-W. Wei, C.-K. Liao, C. Poe, S. Jhan, A.-A. Ding, and Y.-N. Wu "In vivo photoacoustic molecular imaging with simultaneous multiple selective targeting using antibody-conjugated gold nanorods," Optics Express 16, 18605-18615 (2008).

19.R. K. Jain, and T. Stylianopoulos "Delivering nanomedicine to solid tumors," Nature Reviews Clinical Oncol. 7, 653-664 (2010).

20.R. Goel, N. Shah, R. Visaria, G. F. Paciotti, and J. C. Bischof"Biodistribution of tnf-alpha-coated gold nanoparticles in an in vivo model system," Nanomedicine 4, 401-410 (2009).

21.N. Khlebtsov, and L. Dykman "Biodistribution and toxicity of engineered gold nanoparticles: A review of in vitro and in vivo studies," Chem. Soc. Rev. 40, 1647-1671 (2011).

22. Clinicaltrials.gov [Internet] Bethesda: U.S. National Institutes of Health. 2000- [cited 2012 Sept 1]. Available from: http://www.clinicaltrials.gov/.

23.P. K. Jain, K. S. Lee, I. H. El-Sayed, and M. A. El-Sayed "Calculated absorption and scattering properties of gold nanoparticles of different size, shape, and composition: Applications in biological imaging and biomedicine," J. Phys. Chem. B 110, 7238-7248 (2006).

24.C. L. Bayer, Y. S. Chen, S. Kim, S. Mallidi, K. Sokolov, and S. Emelianov "Multiplex photoacoustic molecular imaging using targeted silica-coated gold nanorods," Biomed. Optics Express 2, 1828-1835 (2011).

25.Y.-S. Chen, W. Frey, S. Kim, K. Homan, P. Kruizinga, K. Sokolov, and S. Emelianov "Enhanced thermal stability of silica-coated gold nanorods for photoacoustic imaging and image-guided therapy," Optics Express 18, 88678878 (2010).

26. Y. S. Chen, W. Frey, S. Kim, P. Kruizinga, K. Homan, and S. Emelianov "Silica-coated gold nanorods as photoacoustic signal nanoamplifiers," Nano Lett. 11, 348-354 (2011).

27.S. Kim, Y.-S. Chen, G. P. Luke, and S. Y. Emelianov "In vivo threedimensional spectroscopic photoacoustic imaging for monitoring nanoparticle delivery," Biomed. Optics Express 2, 2540-2550 (2011).

28.S. Kim, Y.-S. Chen, G. P. Luke, and S. Y. Emelianov "In vivo threedimensional spectroscopic photoacoustic imaging for monitoring nanoparticle delivery," Biomed. Optics Express 2, 2540-2550 (2011).

29. S. Prahl "Optical absorption of hemoglobin," (Oregon Medical Laser Center) (1999). 
30.C. Sehgal, S. Weinstein, P. Arger, and E. Conant "A review of breast ultrasound," J. Mammary Gland Biology and Neoplasia 11, 113-123 (2006).

31.A. L. Harris "Hypoxia--a key regulatory factor in tumour growth," Nature Reviews Cancer 2, 38-47 (2002).

32.G. P. Luke, D. Yeager, and S. Y. Emelianov "Biomedical applications of photoacoustic imaging with exogenous contrast agents," Ann. Biomed. Eng. 40, 422-437 (2012).

33.K. H. Song, E. W. Stein, J. A. Margenthaler, and L. V. Wang "Noninvasive photoacoustic identification of sentinel lymph nodes containing methylene blue in vivo in a rat model," J. Biomed. Optics 13, 054033 (2008).

34.K. A. Homan, M. Souza, R. Truby, G. P. Luke, C. Green, E. Vreeland, and S. Emelianov "Silver nanoplate contrast agents for in vivo molecular photoacoustic imaging," ACS Nano 6, 641-650 (2012).

35.J.-W. Kim, E. I. Galanzha, E. V. Shashkov, H.-M. Moon, and V. P. Zharov "Golden carbon nanotubes as multimodal photoacoustic and photothermal high-contrast molecular agents," Nature Nanotechnology 4, 688-694 (2009).

36.D. E. Owens, J. K. Eby, Y. Jian, and N. A. Peppas "Temperatureresponsive polymer-gold nanocomposites as intelligent therapeutic systems," J. Biomed. Mat. Res. Part A 83A, 692-695 (2007).

37.W. F. Zandberg, A. B. S. Bakhtiari, Z. Erno, D. Hsiao, B. D. Gates, T. Claydon, and N. R. Branda "Photothermal release of small molecules from gold nanoparticles in live cells,” Nanomedicine 8, 908-915 (2012).

\section{AGRADECIMIENTOS}

Los autores reconocen el apoyo de los Institutos Nacionales de Salud (NIH) en las subvenciones F32CA159913 (C.L. Bayer), y F31CA168168 (G.P. Luke). Agradecemos a Juili Kevlar por proporcionar las imágenes TEM de nanovara de oro recubierta de sílice, y al Dr. Kimberly Homan por proporcionar las imágenes TEM de placa de plata. También agradecemos a Shailja Tewari de Visualsonics, Christian Wiest de iThera Medical, y a Richard Moss de Endra Life Sciences por proporcionar las imágenes compiladas en la figura 3. 